Fifth International Conference on Sustainable Construction Materials and

Technologies. http://www.claisse.info/Proceedings.htm

\title{
CHARACTERIZING THE PERFORMANCE OF TRANSVERSELY CONFINED MULTI-CULM BAMBOO TO STEEL CONNECTIONS
}

\author{
Nischal P. N. Pradhan ${ }^{1}$, Elias G. Dimitrakopoulos ${ }^{1}$, Themelina S. Paraskeva ${ }^{2}$ \\ ${ }^{1}$ Department of Civil and Environmental Engineering, The Hong Kong University of \\ Science and Technology, Clear Water Bay, Kowloon, Hong Kong
}

${ }^{2}$ School of Engineering, Edith Cowan University, Joondalup WA 6027, Australia

\begin{abstract}
The present research experimentally examines the axial behavior of transversely confined multi-culm bamboo to steel connections, using Kao Jue (Bambusa pervariabilis) bamboo species. The study characterizes under axial monotonic loading, the performance in terms of strength, ductility and failure modes. It then evaluates changes in performance under axial quasi-static reversed cyclic loading. Findings reveal that transverse confinement (through hose-clamps) is highly effective in preventing longitudinal splitting of bamboo culms. The connections exhibit large plastic deformations with sufficient strength and ductility. When compared to the monotonic response, early bolt-fracture hinders the cyclic performance. The connections nevertheless comply with the philosophy of capacity-based design - the ductile components (i.e. the bolts) fail before the brittle components (i.e. the culms). Notably, the adopted European Yield Model can analytically estimate the experimental yield loads with good accuracy. This ultimately indicates a path towards a more rational and engineered design of bamboo structures.
\end{abstract}

Keywords: Steel, confined multi-culm bamboo, transverse confinement.

\section{INTRODUCTION}

Bamboo is among the oldest construction materials. It is eco-friendly and renewable (Escamilla et al. 2018) with remarkable mechanical properties. In areas where it is indigenous, full-culm or round bamboo sees extensive use in traditional housing applications, scaffoldings and bridges (Minke 2012). A more widespread application is however limited, among other reasons, due to the shortage of analytical tools for rational analysis and design (Gatóo et al. 2014; Harries et al. 2012) and also the lack of practical and reliable engineered joints or connection systems (Janssen 2000). This study focuses on the said challenges, and proposes an engineered solution utilizing full-culm bamboo members for truss structures. 
Among the several types of engineered solutions (Disén \& Clouston 2013), this study utilizes connections with metal fasteners (Sassu et al. 2016; Morisco \& Mardjono 1995) due to their ubiquity, versatility and ease of use. The unique geometrical and mechanical properties (Wegst \& Ashby 2007; Amada et al. 1996) and inherent variations in the natural full-culm bamboo (Harries et al. 2017; Sharma et al. 2015) bring challenges to this end. The weak transverse axis makes culms vulnerable to brittle longitudinal splitting (Sharma et al. 2013), which greatly limits the performance of plain-bolted connections. This can be controlled by transversely confining the culms. (Awaludin \& Andriani 2014) for example used FRP wraps with epoxy resin, which brought significant improvements in performance. (Paraskeva et al. 2017) utilized stainless steel hose-clamps to provide confinement and thus increase the resistance to splitting for the culms used in the bamboo footbridge.

The present study examines the performance of transversely confined bamboo to steel bolted connections under both monotonic and quasi-static reversed cycling loading. The motivation originates from the need to provide reliable and practical engineered connections as a sustainable solution for the developing areas, e.g. (Paraskeva et al. 2017). The goal is to characterize experimentally the changes in performance under reverse cyclic (e.g. dynamic) conditions, and thus complement the pertinent findings from the monotonic loading tests.

\section{METHODOLOGY}

Figure 1 shows experimental test specimen with the confined connections. The culms have average external diameter $48.01 \mathrm{~mm}$ (SD 4.34), average wall thickness $6.67 \mathrm{~mm}$ (SD 2.15) and average moisture content of $10.05 \%$ (SD 1.79), where

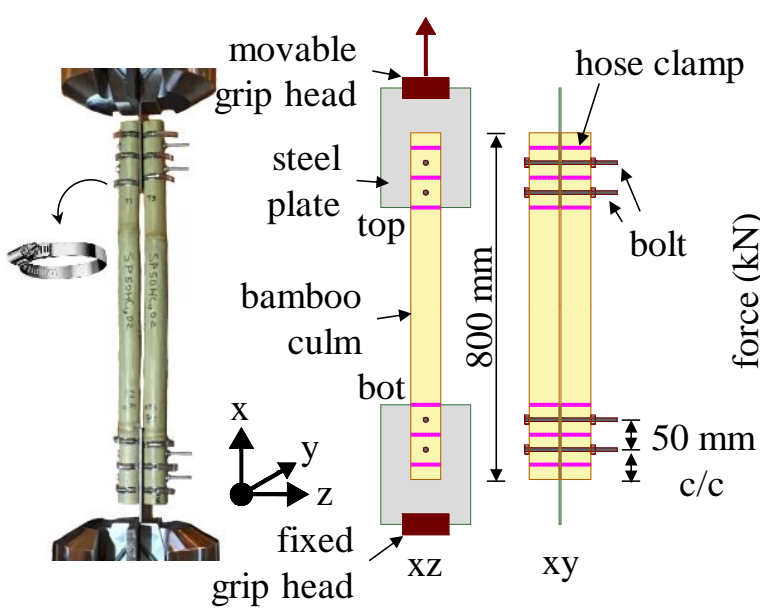

(a) (b)

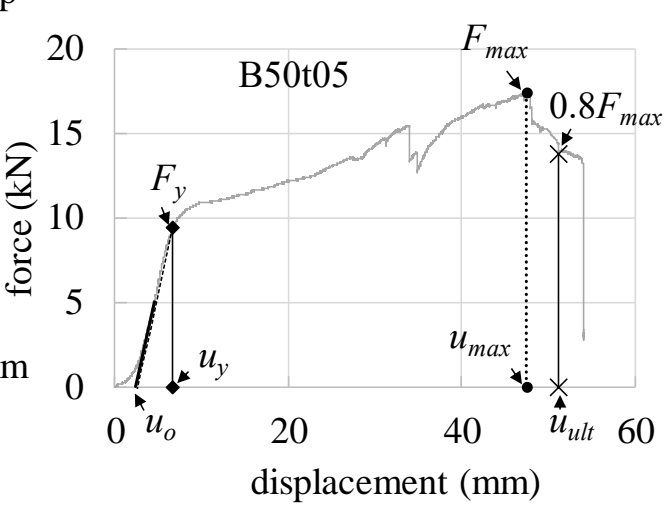

(c)

Figure 1: (a) experimental arrangement of transversely confined (Type B) specimens with (b) detailing; (c) force-displacement plots showing important points.

SD stands for standard deviation. All steel components are of grade SS304, with the 
steel plates thickness of $3 \mathrm{~mm}$ and the hose-clamps of size $40-63 \mathrm{~mm}$. The fullythreaded bolts and the nuts are of type A2-70 and size M6. The oversized bolt-holes in the culm-walls and steel plates provide $1 \mathrm{~mm}$ of clearance. The center-to-center bolt-spacing and the end-lengths are at $50 \mathrm{~mm}$ distance. The hose-clamps installed at approximately $25 \mathrm{~mm}$ center-to-center away from either side of the bolts provide the transverse confinement to the culms.

All tests were conducted at the Structural Engineering Laboratory of The Hong Kong University of Science and Technology using the MTS 810 universal testing machine. The nomenclature used for the specimens is similar to that in (Paraskeva et al. 2019) starting with "B" for transversely confined connections followed by "50t", "c" or subscript "cl" for monotonic tension loading, monotonic compression loading or cyclic loading respectively, and ending with the specimen numbers 01 to 05 . For monotonic tests, the rate of loading is set to $0.01 \mathrm{~mm} / \mathrm{s}$. The cyclic test uses (ISO 16670:2003(E)) displacement controlled loading protocol. The reference displacement $\Delta_{\mathrm{m}}$ for the loading protocol is the failure displacement $u_{u l t}$ at $80 \%$ of maximum load or the load-carrying capacity $F_{\max }$ (e.g. Figure $1 \mathrm{c}$ ) of the monotonic load-displacement response. This study adopts $44.52 \mathrm{~mm}$ as $\Delta_{\mathrm{m}}$ for Type $\mathrm{B}_{\mathrm{cl}}$ specimens. The rate of loading for cyclic tests is $0.5 \mathrm{~mm} / \mathrm{s}$, with tension intervals preceding the compression intervals.

\section{RESULTS}

\section{Failure Modes}

The transverse confinement from hose-clamps prevents premature

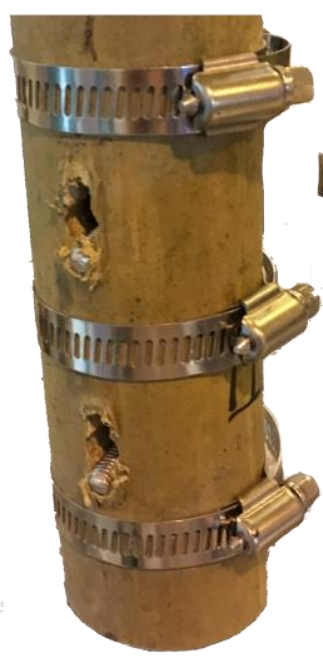

(a)

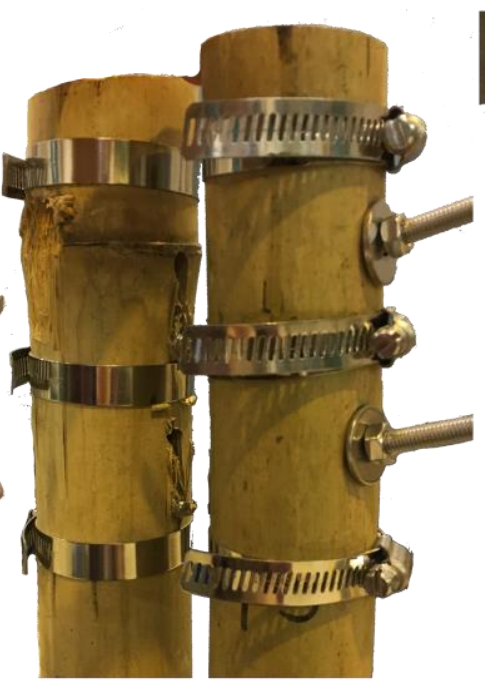

(b)

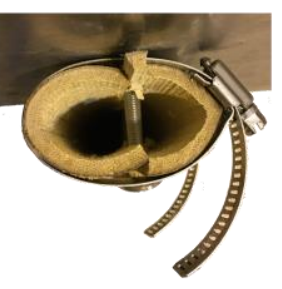

(c)

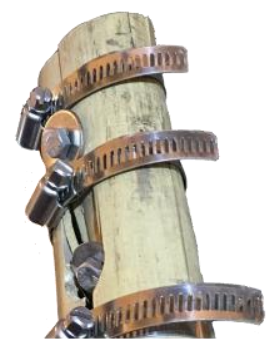

(d)

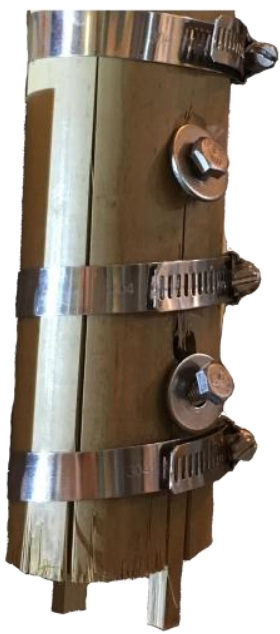

(e)

Figure 2: bolt-embedment and fracture in (a) cyclic specimen and (b) monotonic specimen; (c) crushing by ovalization; (d) bolt pull-through (with crushing); and (e) row-shearing (with crushing). 
culm-failure by brittle longitudinal splitting and allows extended plastic deformation and embedment of the bolts into the culms (Figure $2 \mathrm{a}$ and $\mathrm{b}$ ). The culm-walls adjacent to the steel plates sustain greater embedment damage compared to the outward facing culm-walls, and forms larger cavities (Figure 2b, left culm) around bolt vicinity. The deforming bolts transversely compress and ovalize the culm-ends. For B50t specimens, this results in culm-damage by crushing (Figure 2c) due to excessive ovalization of culm cross-section and/or row-shearing (Figure 2e), with corresponding loss in strength (at $30 \mathrm{~mm}$ displacements, Figure 3a). For three specimens B50t01-03, this point corresponds to their maximum developed load (or the load-carrying capacity) and thus have reached failure. The remaining B50t04 and 05 specimens fail by bolt-fracture. The bolt-heads and the nuts can also penetrate through the culm walls (e.g. specimens B50t01 and B50t03, Figure 2d). Bc specimens are resistant to brittle culm-damage by crushing/row-shearing of the culm-ends and fail by bolt-fracture (e.g. Figure $2 b$ ). The examined cyclic $\mathrm{B}_{\mathrm{cl}}$ specimens fail by early bolt-fracture (e.g. Figure 2a) that occurs before sustaining brittle culm-damage (i.e. the examined specimens do not show failure modes Figure 2c-e).

\section{Axial Response}

Figure 3 and Figure 4 show the results of monotonic and cyclic load tests respectively. The maximum load $F_{\max }$ (Figure 5a) of the load-displacement curve/envelope denotes the load-carrying capacity, while $u_{\max }$ (Figure $5 \mathrm{~b}$ ) describes the displacement level corresponding to $F_{\max }$. The envelope curves are superimposed on the respective cyclic curves in the same plot (Figure 4). Both Figure 3 and 4 also provide the specimen cross-sectional area $A_{s}$ by averaging the total cross-sectional area at the ends of the two component culms (where the culm diameters and thicknesses as per (ISO 22157-1:2004(E)) are known). The normalized load-carrying capacity of the specimens $f_{n}$ is $F_{\max }$ divided by the mean (total) cross-sectional area of the culms $A_{s}$, i.e., $f_{n}=F_{\max } / A_{s}$. The $f_{n}$ (Figure 5c) indicates the maximum axial stress present within the culms. The pertinent

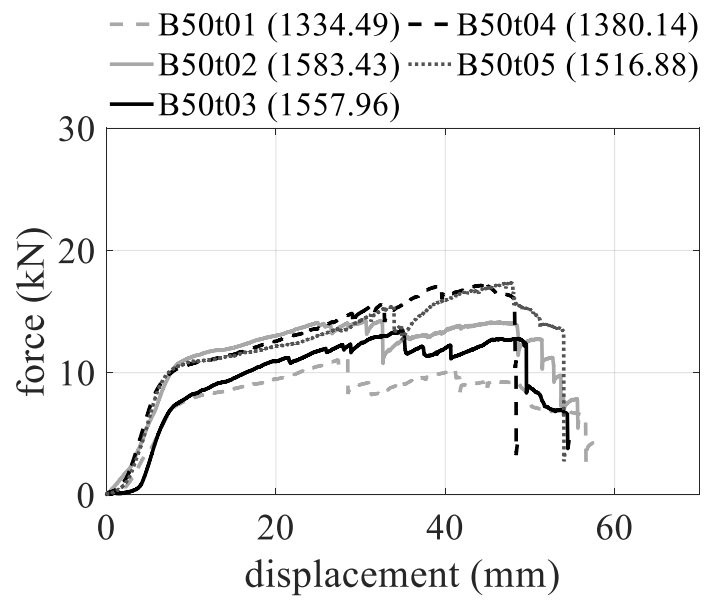

(a)

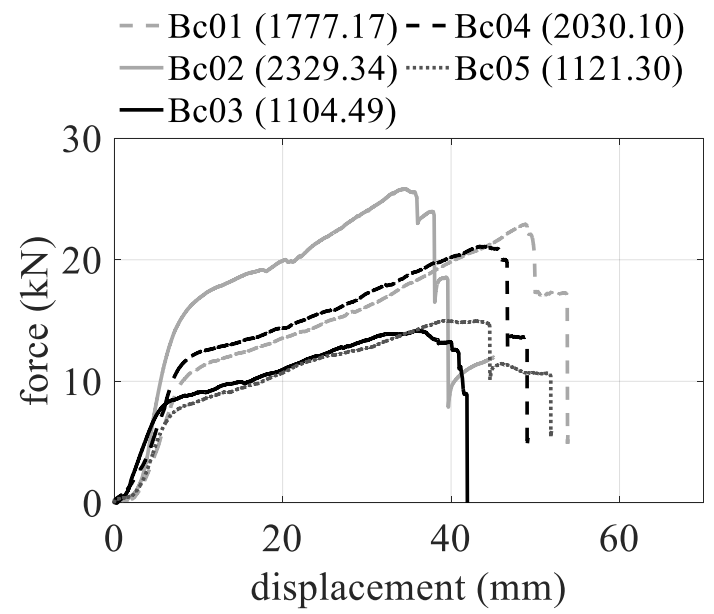

(b)

Figure 3: (a) B50t (tension) specimens (b) Bc (compression) specimens; values within brackets provide the cross-sectional area of the specimens 


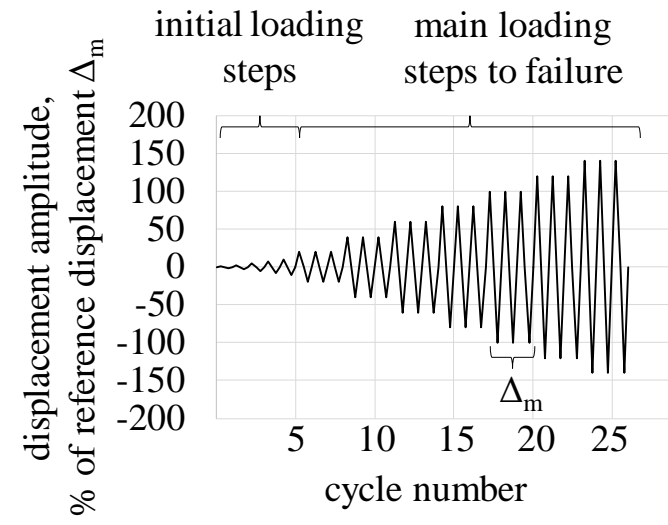

(a)

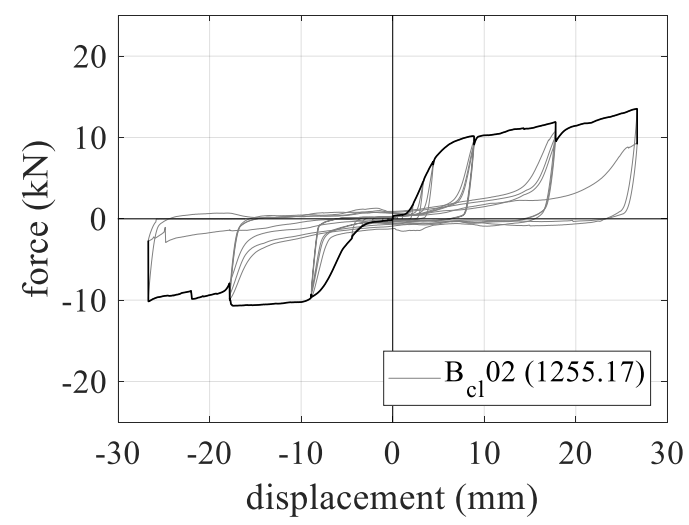

(c)

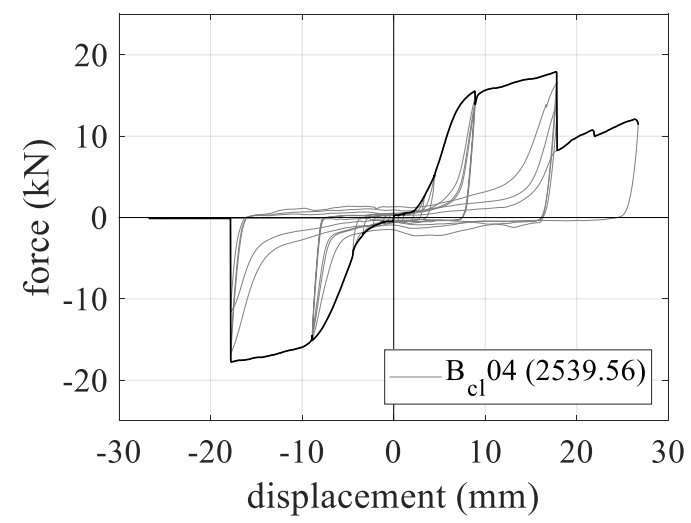

(e)

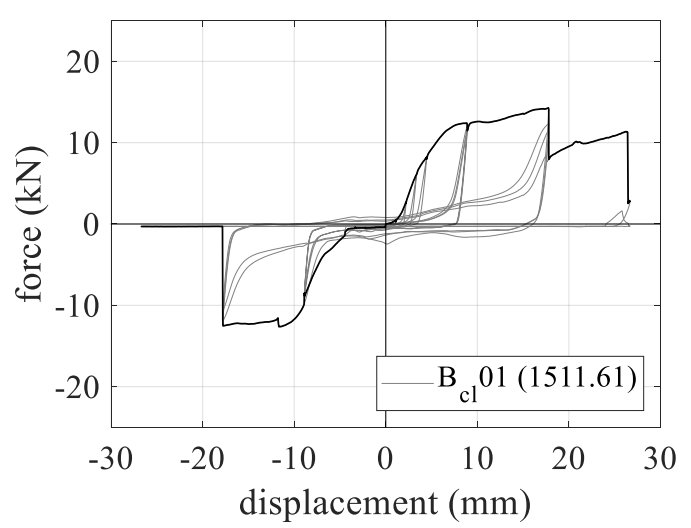

(b)

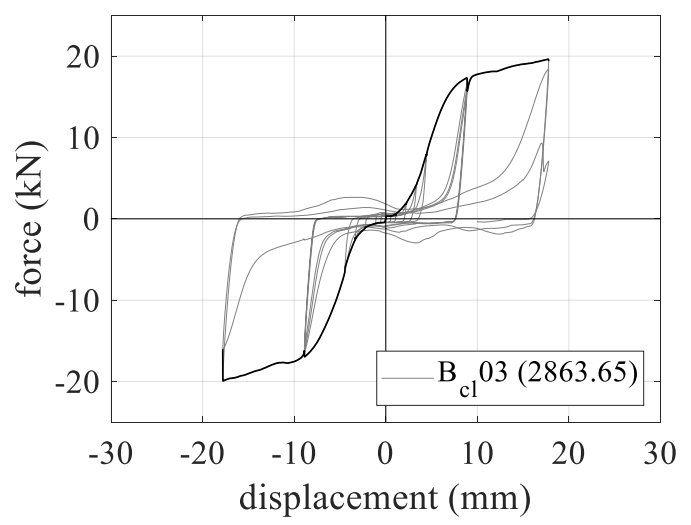

(d)

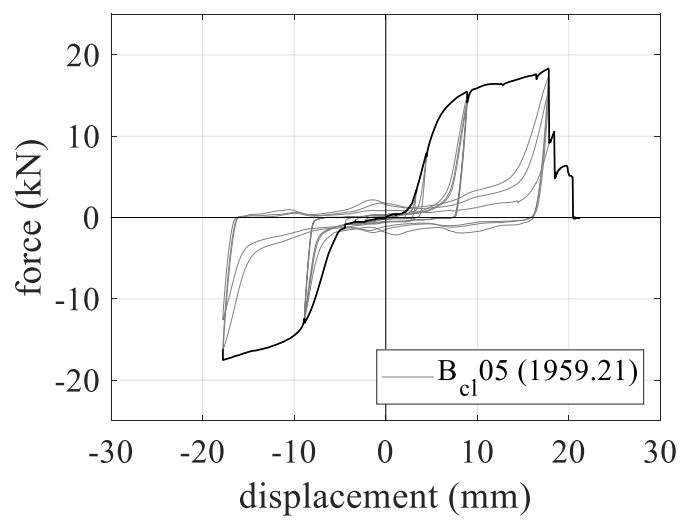

(f)

Figure 4: (a) ISO 16670:2003(E) loading protocol; (b) to (f) force-displacement response of the $\mathrm{B}_{\mathrm{cl}}$ specimens, superimposed by the envelope curves (black-line) and mean cross-sectional areas within brackets

normalized coefficients of variation $\hat{C}_{V}=\left(S D / \bar{f}_{n}\right) \times 100 \%$, where SD stands for standard deviation and $\bar{f}_{n}$ the mean of normalized load-carrying capacities. The analysis also adopts the relative strength indicator $\lambda_{t}=\left(f_{n} / f_{t}\right) \times 100 \%$ under 


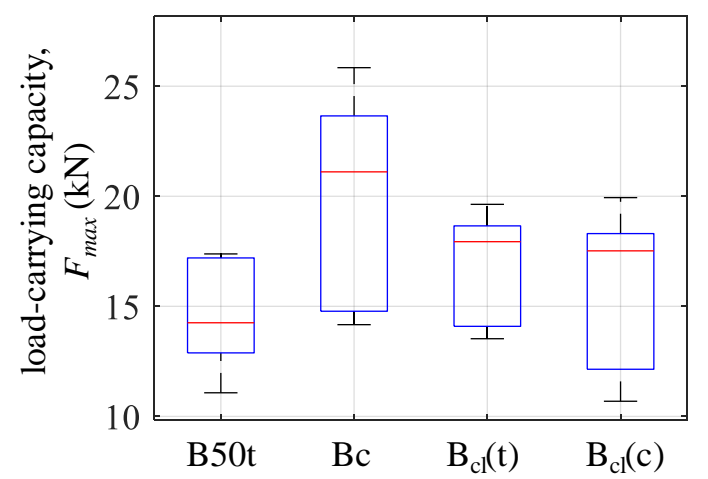

(a)

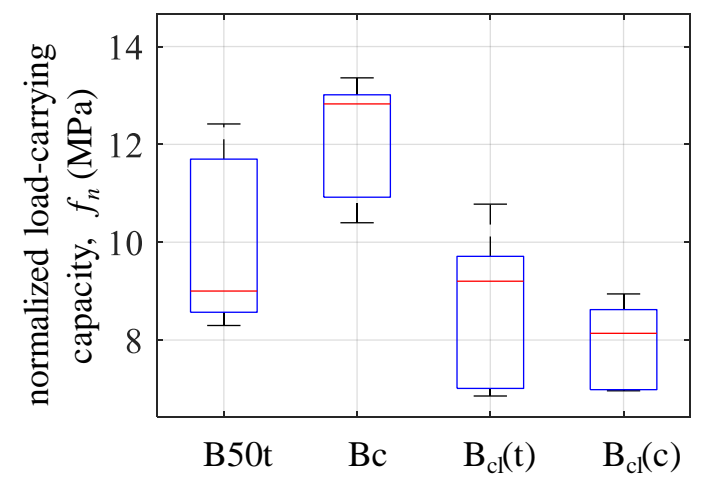

(c)

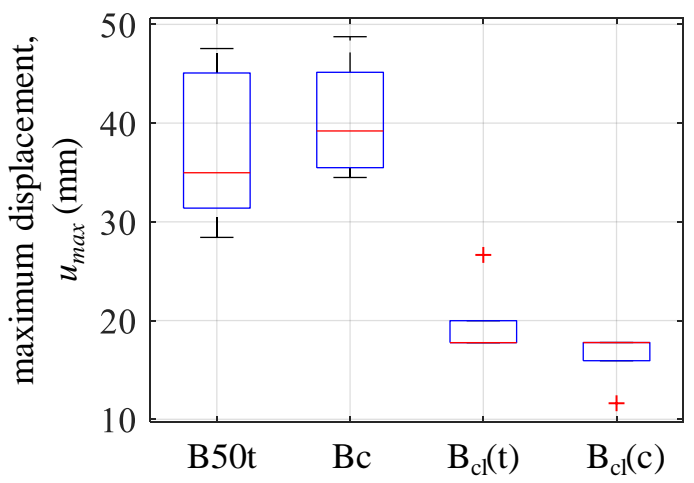

(b)

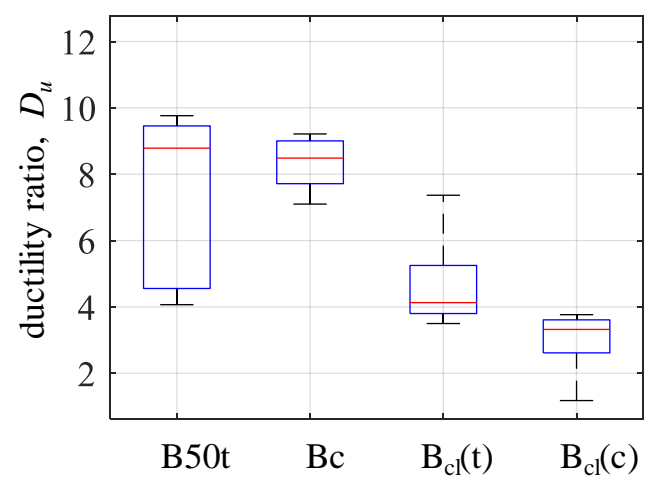

(d)

Figure 5: Box-plots showing (a) load-carrying capacity, (b) maximum displacement of the examined specimens, (c) normalized load-carrying capacity, and (d) ductility ratio of the examined specimens and tension/compression envelopes.

tension and $\lambda_{c}=\left(f_{n} / f_{c}\right) \times 100 \%$ under compression as a measure of efficiency. The compressive and tensile strength of the Kao Jue bamboo culms are $f_{c}=44.56 \mathrm{MPa}$ and $f_{t}=134.05 \mathrm{MPa}$ respectively (Paraskeva et al. 2017). The ductility ratio obtained also from the force-displacement curves (Figure 3) and envelopes (Figure 4) is $D_{u}=$ $\left(u_{\max }-u_{o}\right) /\left(u_{y}-u_{o}\right)$ (Figure $\left.5 \mathrm{~d}\right)$. In other words, the $D_{u}$ is the ratio of displacement at maximum load $u_{\max }$ to the yield displacement $u_{y}$ ((Jorissen \& Fragiacomo 2011), see Figure 1c), herein adjusted with the initial displacement $u_{o}$.

The examined B50t specimens attain a mean load-carrying capacity $\bar{F}_{\max }=14.67 \mathrm{kN}$ (SD 2.64) at the corresponding mean displacement $\bar{u}_{\max }=34.52 \mathrm{~mm}$ (SD 8.09). The normalized load-carrying capacity is $f_{n}=9.97 \mathrm{~N} / \mathrm{mm}^{2}$ (SD 1.85) (Figure 5c) with an average ductility ratio $\bar{D}_{u}$ of 7.34 (SD 2.72) (Figure 5d) and the efficiency $\bar{\lambda}_{t}=\left(f_{n} / f_{t}\right) \times 100 \%=7.44 \%$ (SD 1.38) (Figure 6b). When compared to the plain specimens (Paraskeva et al. 2019), transverse confinement inhibits premature failure by longitudinal splitting of culms and significantly improves the performance. 
(a)

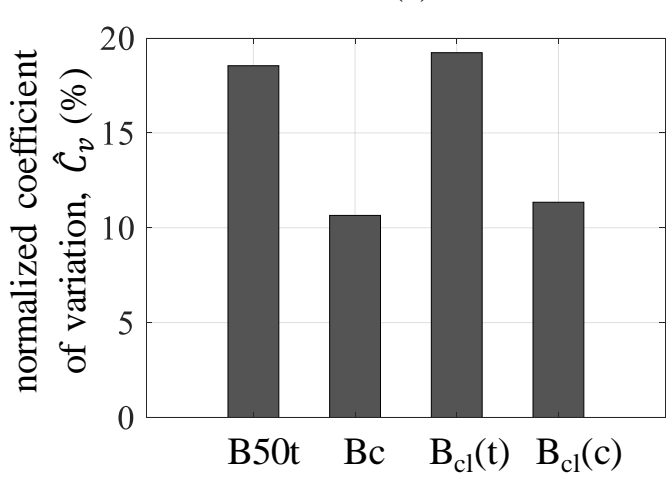

(b)

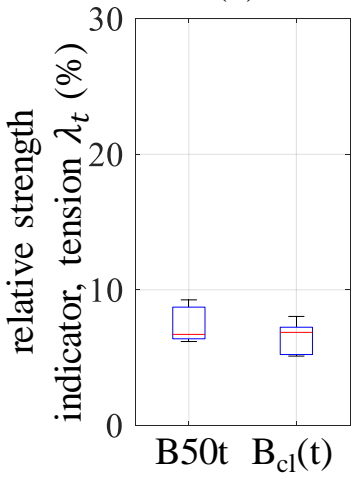

(c)

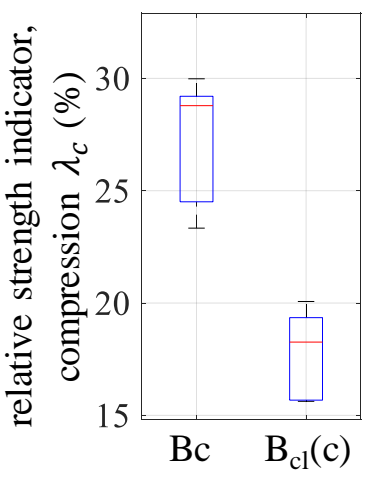

Figure 6: (a) normalized coefficient of variation; box-plots for (b) relative strength indicator (tension), (c) relative strength indicator (compression)

The examined Bc specimens develop a mean load-carrying capacity $\bar{F}_{\max }=19.80 \mathrm{kN}$ (SD 5.07) (Figure 5a) at the corresponding displacement $\bar{u}_{\max }=40.44 \mathrm{~mm}$ (SD 5.91). The normalized load-carrying capacity is $f_{n}=12.12 \mathrm{~N} / \mathrm{mm}^{2}$ (SD 1.29) with an average ductility ratio $\bar{D}_{u}$ of 8.33 (SD 0.84) and the efficiency $\bar{\lambda}_{c}=\left(f_{n} / f_{c}\right) \times 100 \%=$ $27.19 \%$ (SD 2.90) (Figure 6c). The Bc specimens are more resistant to culm-damage and their performance is marginally greater than that of B50t specimens.

Figure 5-6 also presents the performance of $B_{\mathrm{cl}}$ specimens subjected to quasi-static reversed cyclic loading (Figure 4a). Under tension intervals, the examined $\mathrm{B}_{\mathrm{cl}}$ specimens achieve a mean load-carrying capacity $\bar{F}_{\max }=16.74 \mathrm{kN}$ (SD 2.68) at mean displacement $\bar{u}_{\max }=19.54$ (SD 3.97). The normalized load-carrying capacity is $f_{n}=$ $8.65 \mathrm{~N} / \mathrm{mm}^{2}$ (SD 1.66) with an average ductility ratio $\bar{D}_{u}=4.69$ (SD 1.54) and the efficiency $\bar{\lambda}_{t}=6.45 \%$ (SD 1.24). Under compression intervals, the specimens display $\bar{F}_{\max }=15.71 \mathrm{kN}(\mathrm{SD} 3.88)$ at $\bar{u}_{\max }=16.46$ (SD 2.71). The normalized load-carrying capacity is $f_{n}=7.91 \mathrm{~N} / \mathrm{mm}^{2}$ (SD 0.90) with an average ductility ratio $\bar{D}_{u}=2.98$ (SD 1.04) and the efficiency $\bar{\lambda}_{c}=17.75 \%$ (SD 2.01).

The examined envelopes of $\mathrm{B}_{\mathrm{cl}}$ specimens show lower performance when compared to the respective response of $\mathrm{B} 50 \mathrm{t}$ and $\mathrm{Bc}$ specimens. The tension envelopes display $\bar{F}_{\max }$ at roughly $48 \%$ lower $\bar{u}_{\max }$ than the monotonically loaded B50t specimens. The $\bar{f}_{n}$ reduces by $13 \%$ while the $\bar{D}_{u}$ reduces by $36 \%$. As the connections sustain damage in the preceding tension intervals, the performance reduction during compression intervals is more severe. Specifically, the compression envelopes attain $\bar{F}_{\max }$ at roughly $59 \%$ lower $\bar{u}_{\max }$ than the Bc specimens. The $\bar{f}_{n}$ reduces by $35 \%$

Table 1: Geometry details used for EYM calculation, $L_{s}$ is the external diameter, $t_{w s}$ 
is the culm-wall thickness.

\begin{tabular}{ccc|ccc|ccc}
\hline specimen & $\begin{array}{c}L_{s} \\
\mathrm{~mm}\end{array}$ & $\begin{array}{c}t_{w s} \\
\mathrm{~mm}\end{array}$ & specimen & $\begin{array}{c}L_{s} \\
\mathrm{~mm}\end{array}$ & $\begin{array}{c}t_{w s} \\
\mathrm{~mm}\end{array}$ & specimen & $\begin{array}{c}L_{s} \\
\mathrm{~mm}\end{array}$ & $\begin{array}{c}t_{w s} \\
\mathrm{~mm}\end{array}$ \\
\hline $\mathrm{B} 50 \mathrm{t} 01$ & 45.05 & 5.04 & $\mathrm{Bc} 01$ & 49.86 & 6.21 & $\mathrm{~B}_{\mathrm{cl}} 01$ & 44.96 & 6.74 \\
$\mathrm{~B} 50 \mathrm{t} 02$ & 55.90 & 5.41 & $\mathrm{Bc} 02$ & 47.68 & 9.18 & $\mathrm{~B}_{\mathrm{cl}} 02$ & 46.18 & 4.54 \\
$\mathrm{~B} 50 \mathrm{t} 03$ & 47.27 & 5.74 & $\mathrm{Bc} 03$ & 42.37 & 4.51 & $\mathrm{~B}_{\mathrm{cl}} 03$ & 55.20 & 8.73 \\
$\mathrm{~B} 50 \mathrm{t} 04$ & 43.35 & 5.57 & $\mathrm{Bc} 04$ & 49.97 & 7.09 & $\mathrm{~B}_{\mathrm{cl}} 04$ & 52.38 & 7.38 \\
$\mathrm{~B} 50 \mathrm{t} 05$ & 49.00 & 5.31 & $\mathrm{Bc} 05$ & 41.09 & 5.08 & $\mathrm{~B}_{\mathrm{cl}} 05$ & 47.10 & 7.40 \\
\hline
\end{tabular}

while the $\bar{D}_{u}$ reduces by $64 \%$. The normalized coefficient of variation is similar between monotonic and cyclic specimens (Figure 6a).

\section{Connection Yield Loads}

This section adopts the European Yield Model (EYM) (Parsons 2001; Technical Report 12 2015), implemented in multi-culm bamboo to steel connections by (Paraskeva et al. 2019). The EYM describes multiple possible modes of yielding that depends upon the geometry of connection components (see Table 1 and methodology section), embedment strength of bamboo and the yield moment of the bolts. The pertinent values of embedment strength of the culms for Mode IIIs (hollow section) $f_{e s}=51.99 \mathrm{MPa}$ (Paraskeva et al. 2019), while the yield moment of the bolts $M_{y}=$ 14880.54 Nmm (Paraskeva et al. 2019).

All Type B specimens display a yielding mode resembling the EYM Mode IIIs hollow-section yielding. The analytical yield load of a connection is twice the Mode IIIs (hollow section) yield load of a single bolt. Figure 7 compares the yield loads of the connections predicted analytically by the EYM to that determined experimentally using the 5\% offset method (F1575-03 2003). For cyclic specimens, the study takes $5 \%$ offsets from tension envelopes. According to the

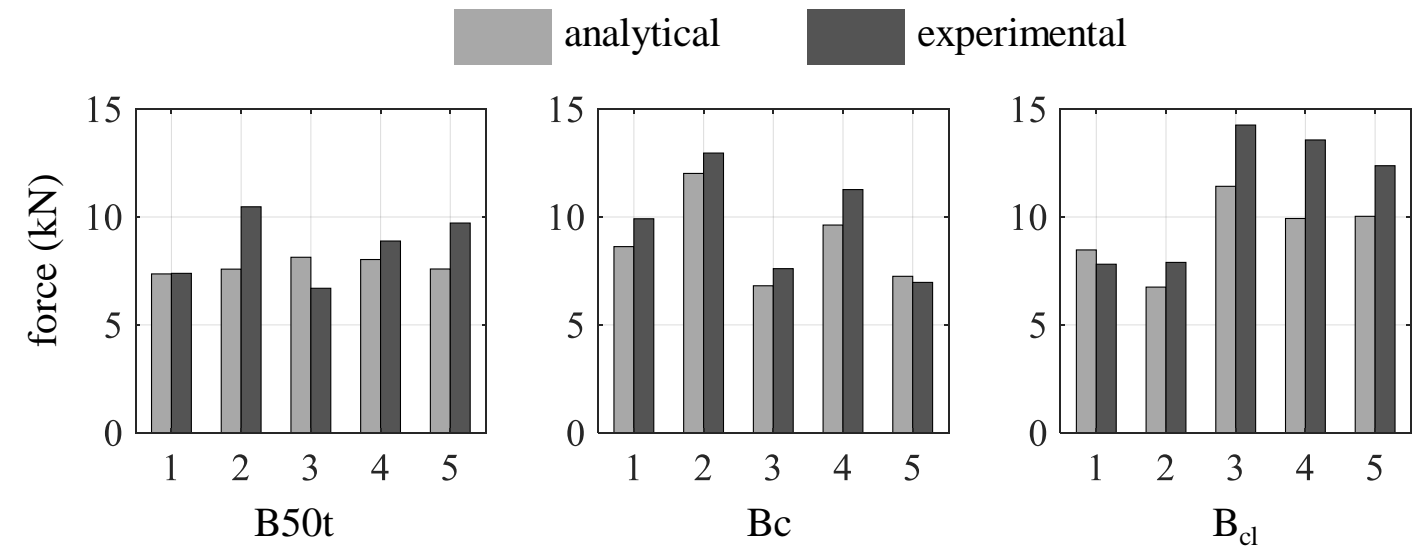

Figure 7: Analytical vs Experimental yield loads for the examined specimens 
adopted loading protocol (Figure 4a), the bolts yield for the first time during the tension intervals as they precede the compression intervals. For B50t specimens, the average analytical yield load $\bar{F}_{a}=7.74 \mathrm{kN}$ underestimates the experimental yield load $\bar{F}_{y}=$ $8.63 \mathrm{kN}$ by $10.3 \%$. Similarly for the Bc specimens, the $\bar{F}_{a}=8.87 \mathrm{kN}$ underestimates the experimental $\bar{F}_{y}=9.75 \mathrm{kN}$ by $9.0 \%$. For the $\mathrm{B}_{\mathrm{cl}}$ specimens, the mean analytical $\bar{F}_{a}=9.32 \mathrm{kN}$ underestimates the mean experimental $\bar{F}_{y}=11.18 \mathrm{kN}$ by $16.6 \%$. Overall, the adopted equations can analytically predict the experimental yield load of a connection with good accuracy.

\section{CONCLUSION}

The transversely confined connections (Type B) resist inhibit premature failure by longitudinal splitting of the bamboo culm. This allows the connections to enter an extended plastic state through predictable ductile yielding of bolts. In particular, the adopted European Yield Model can analytically estimate the experimental yield load with satisfying accuracy. Compared to the monotonic response however, early boltfracture reduces ductility and limits the cyclic performance. The current configuration of Type B connections is nevertheless in accordance with the philosophy of capacitybased design. The ductile yielding and the pertinent values of relative strength indicators ensure the associated culm member elements (which can undergo brittle catastrophic compression/tension failures) remain safely within the elastic state. This finding however, also suggests ample headroom for further improvement in design and efficiency of the connections.

\section{REFERENCES}

Amada, S. et al., 1996. The Mechanical Structures of Bamboos in Viewpoint of Functionally Gradient and Composite Materials. Journal of Composite Materials, 30(7), pp.800-819.

Awaludin, A. \& Andriani, V., 2014. Bolted bamboo joints reinforced with fibers. Procedia Engineering, 95, pp.15-21. Available at: http://dx.doi.org/10.1016/j.proeng.2014.12.160.

Disén, K. \& Clouston, P.L., 2013. Building with bamboo: a review of culm connection technology. Journal of Green Building, 8(4), pp.83-93.

Escamilla, E.Z. et al., 2018. Industrial or traditional bamboo construction? Comparative life cycle assessment (LCA) of bamboo-based buildings. Sustainability (Switzerland), 10(9).

F1575-03, 2003. Standard Test Method for Determining Bending Yield Moment of Nails. West Conshohocken, PA: ASTM International, West Conshohocken, PA. Available at: http://www.astm.org/cgi-bin/resolver.cgi?F1575.

Gatóo, A. et al., 2014. Sustainable structures: bamboo standards and building codes. Proceedings of the Institution of Civil Engineers - Engineering Sustainability, 167(5), pp.189-196. 
Harries, K.A. et al., 2017. Geometric and material effects on bamboo buckling behaviour. Proceedings of the Institution of Civil Engineers - Structures and Buildings, 170(4), pp.236-249. Available at: http://www.icevirtuallibrary.com/doi/10.1680/jstbu.16.00018.

Harries, K.A., Sharma, B. \& Richard, M., 2012. Structural Use of Full Culm Bamboo: The Path to Standardization. International Journal of Architecture, Engineering and Construction, 1(2), pp.66-75.

ISO 16670:2003(E), 2003. Timber structures — joints made with mechanical fasteners - quasi-static reversed-cyclic test method. Switzerland: International Organization for Standardization, Geneva, Switzerland.

ISO 22157-1:2004(E), 2004. Bamboo - Determination of physical and mechanical properties - Part 1: Requirements. Switzerland: International Organization for Standardization, Genveva, Switzerland.

Janssen, J.J.A., 2000. Technical Report 20: Designing and building with bamboo, International Network for Bamboo and Rattan.

Jorissen, A. \& Fragiacomo, M., 2011. General notes on ductility in timber structures. Engineering Structures, 33(11), pp.2987-2997. Available at: http://dx.doi.org/10.1016/j.engstruct.2011.07.024 [Accessed May 5, 2018].

Minke, G., 2012. Building with Bamboo, Birkhäuser, Basel.

Morisco \& Mardjono, F., 1995. Strength of filled bamboo joint. In I. V. R. Rao et al., eds. Proceedings of the Vth International Bamboo Workshop and the IV International Bamboo Congress. Bali , Indonesia: International Development Research Centre.

Paraskeva, T. et al., 2019. Monotonic loading testing and characterization of new multi-full-culm bamboo to steel connections. Construction and Building Materials, 201, pp.473-483.

Paraskeva, T.S., Grigoropoulos, G. \& Dimitrakopoulos, E.G., 2017. Design and experimental verification of easily constructible bamboo footbridges for rural areas. Engineering Structures, 143, pp.540-548.

Parsons, W.R., 2001. Energy-based modelling of dowel-type connections in woodplastic composite hollow sections. Washington State University.

Sassu, M. et al., 2016. Structural solutions for low-cost bamboo frames: Experimental tests and constructive assessments. Materials, 9(5).

Sharma, B. et al., 2015. Engineered bamboo: state of the art. Proceedings of the Institution of Civil Engineers - Construction Materials, 168(2), pp.57-67. Available at: http://www.icevirtuallibrary.com/doi/10.1680/coma.14.00020.

Sharma, B., Harries, K.A. \& Ghavami, K., 2013. Methods of determining transverse mechanical properties of full-culm bamboo. Construction and Building Materials, 38, pp.627-637.

Technical Report 12, 2015. General Dowel Equations for Calculating Lateral Connection Values with Appendix A. Leesburg, VA: American Wood Council (AWC), Leesburg, VA.

Wegst, U.G.K.K. \& Ashby, M.F., 2007. The structural efficiency of orthotropic stalks, stems and tubes. Journal of Materials Science, 42(21), pp.9005-9014. 